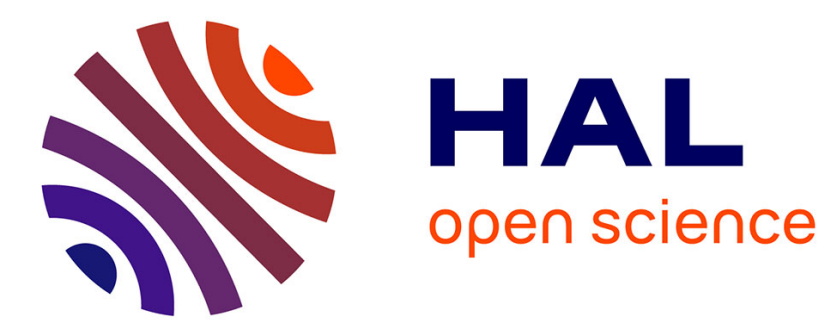

\title{
Simulation of Atmospheric Boundary Layer for Air Inlet Testing in Crossflow
}

\author{
Sylvain Mouton
}

\section{To cite this version:}

Sylvain Mouton. Simulation of Atmospheric Boundary Layer for Air Inlet Testing in Crossflow. AIAA Journal, 2021, 59 (7), pp.2603-2612. 10.2514/1.J059940 . hal-03241049

\section{HAL Id: hal-03241049 \\ https://hal.science/hal-03241049}

Submitted on 28 May 2021

HAL is a multi-disciplinary open access archive for the deposit and dissemination of scientific research documents, whether they are published or not. The documents may come from teaching and research institutions in France or abroad, or from public or private research centers.
L'archive ouverte pluridisciplinaire HAL, est destinée au dépôt et à la diffusion de documents scientifiques de niveau recherche, publiés ou non, émanant des établissements d'enseignement et de recherche français ou étrangers, des laboratoires publics ou privés. 


\title{
Simulation of Atmospheric Boundary Layer for Air Inlet Testing in Crossflow
}

\author{
Sylvain Mouton* \\ ONERA-The French Aerospace Lab, F-31410 Mauzac, France
}

https://doi.org/10.2514/1.J059940

\begin{abstract}
Turbulence grids replicating the velocity profile (including the high-frequency part of the turbulence spectrum) of the atmospheric boundary layer (ABL) have been designed, manufactured, and tested in ONERA wind tunnels. These ABL grids are intended to improve the experimental simulation of the flow entering air inlets of aircraft engines while operating in a crosswind. It was found that the grids properly reproduced the features of the ABL flow that are relevant to this application. A test with an air inlet has confirmed that the grids can have a noticeable effect on the operating range of the air inlet and are therefore of significant interest to engine manufacturers.
\end{abstract}

\section{Nomenclature}

$C_{p} \quad=$ pressure coefficient

$D \quad=$ diameter of air inlet

$d \quad=$ diameter of grid rods

$H \quad=$ height of wind-tunnel channel

$h \quad=$ height above ground

$K=$ local resistance coefficient of the grid

$k \quad=\quad$ spatial frequency of turbulent eddies (1/wavelength)

$l_{u} \quad=\quad$ integral length of turbulent velocity fluctuations $u$

$M \quad$ = local distance between two adjacent grid rod centerlines

$\mathcal{M}=$ Mach number

$p \quad=$ static pressure

$S_{u u}, S_{v v}, S_{w w}=$ power spectral densities of turbulent velocity fluctuations $u, v$, and $w$

$T_{u}, T_{v}, T_{w} \quad=\quad$ turbulence ratios of velocity fluctuations $u, v$ and $w$ with respect to local velocity $U$; $T_{u}=\sigma_{u} / U, T_{v}=\sigma_{v} / U$, and $T_{w}=\sigma_{w} / U$

$U, V, W \quad=\quad$ mean velocities along $x, h$, and $z$, respectively

$u, v, w \quad=\quad$ components of turbulent velocity fluctuations along $x, h$, and $z$, respectively

$x, z=$ longitudinal and transverse coordinates

$\alpha \quad=\quad$ refraction coefficient of the grid

$\zeta \quad=\quad$ local grid solidity (blocked area/total area)

$\lambda \quad=$ model scaling factor

$\rho \quad=$ air density

$\sigma_{u}^{2}, \sigma_{v}^{2}, \sigma_{w}^{2} \quad=\quad$ variances of velocity fluctuations $u, v$, and $w$, respectively

\section{Subscripts}

A

c

fan

Q

$\mathrm{R}$

0

10
$=$ Prandtl antenna in the tunnel

$=$ inlet center

$=$ flow conditions averaged across grid channel height

$=$ ground roughness

$=$ grid parameters averaged across the grid height

$=$ wind reference height $h_{10}=10 \mathrm{~m}$ at full scale
$=$ fan plane in the inlet

Presented at Paper 2017-4132 at the 33rd AIAA Aerodynamic Measurement Technology and Ground Testing Conference, Denver, CO, June 5-9, 2017; received 22 July 2020; revision received 23 November 2020; accepted for publication 7 January 2021; published online 24 May 2021. Copyright $\odot 2021$ by the authors. Published by the American Institute of Aeronautics and Astronautics, Inc., with permission. All requests for copying and permission to reprint should be submitted to CCC at www .copyright.com; employ the eISSN 1533-6794 to initiate your request. See also AIAA Rights and Permissions www.aiaa.org/randp.

*Assistant Director for Scientific and Technical Developments, Wind Tunnel Division/Le Fauga-Mauzac Wind Tunnels Department.

\section{Introduction}

$\mathbf{T}$ HE air inlet of aircraft aeroengines is designed to provide the fan with a steady uniform flow in order to ensure the safe, stable operation of the engine [1,2]. This is particularly challenging to achieve in the case of an air inlet operating in crossflow close to the ground, as is the case at the start of a takeoff roll with a strong crosswind present [3] Indeed, in this case, the airstream captured by the engine has to flow around the small leading-edge radius of the intake lip. This causes the airflow to experience a large local acceleration followed by a steep deceleration. These conditions may cause the boundary layer on the lip to separate, therefore creating large flow distortions at fan plane [4,5]. This phenomenon is difficult to numerically simulate in a reliable manner [6]. The situation is further complicated by the frequent formation of a ground vortex ingested by the inlet [7].

The previously described situation is sketched on Fig. 1a for a stationary aircraft in a $90 \mathrm{deg}$ crosswind: the aircraft is sitting on the ground and immerged in the atmospheric boundary layer (ABL). Previously, this situation was simulated in a wind tunnel, as sketched on Fig. 1b with only the inlet of the engine nacelle represented, with the ground represented by a physical panel and the atmospheric wind generated by the wind-tunnel flow. This crosswind setup has been routinely used in the ONERA F1 wind tunnel, which is a continuous low-speed pressurized (up to 3.85 atmospheres) wind tunnel optimized for high-lift performance testing of civil transport aircraft [8]. This wind tunnel is also used for the test of air inlets at high Reynolds numbers. The required mass flow is generated by venting in short bursts, the pressurized tunnel to atmosphere through the inlet model $[9,10]$.

Comparing Figs. $1 \mathrm{a}$ and $1 \mathrm{~b}$, it is obvious that this experimental setup does not simulate the natural wind in ground effect: the mean flow is uniform (deviations from mean flow well below $0.5 \%$ [8]) instead of reproducing the ABL profile, and the flow has a near-zero turbulence level ( $T_{u}$ below $0.1 \%$ [8]) instead of the highly turbulent $\mathrm{ABL}$, as needed for conventional aircraft testing.

Simulating the ABL flow in a wind tunnel has been a topic of interest to scientists and engineers for a long time. The objective is to better predict the effect of the natural wind in ground effect on objects, e.g., structure loads, flow distortions, or pollutant dispersal $[11,12]$. In many cases (for example, in buildings' aerodynamics), the length scale of the object under study is of the same order of magnitude as the turbulence integral length scales. In that case, the simulation of most of the turbulence spectrum is required in the windtunnel experiment $[11,12]$. This is currently frequently achieved in dedicated wind tunnels fitted with large-scale obstacles (called "obstructions" [13], "wedges" [14], or "spires" [15]) at the entrance of the test section, followed by roughness elements placed on the floor [11]. Suitable wind tunnels feature long test sections to allow the vorticity, locally shed in the flow by the obstacles, to diffuse and turn into the expected homogeneous turbulence. Typically, the length of 


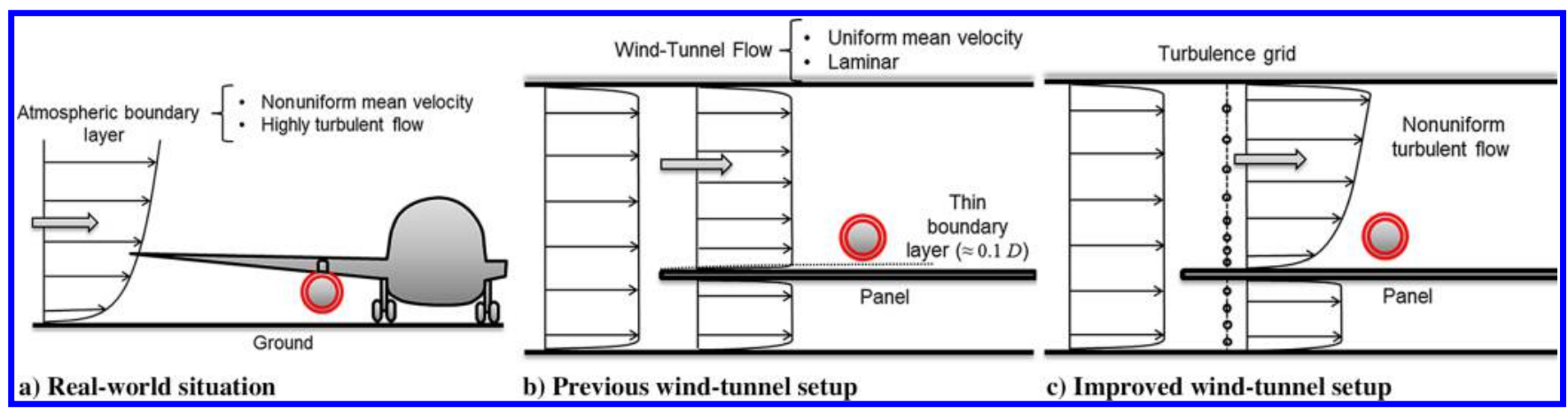

Fig. 1 Wind-tunnel setups for the study of air inlet in crossflow.

the test section is 7 to 10 times its height, although distances as short as 2.6 times the height have been attempted [16], but without reporting measurements directly in the wakes of the spires.

In this work, we chose to replicate only the high-frequency (small turbulent eddies, corresponding to the microscale of Ref. [12]) part of the ABL turbulence spectrum for reasons that will be discussed in Sec. II.B. This reduced requirement made it possible to consider other types of ABL-generating devices (as discussed in Ref. [13]) that do not require a long test section ahead of the model under test. These devices were appropriate for use in the existing wind-tunnel setup of Fig. $1 \mathrm{~b}$ with a distance of only about 1.2 times the test section height available downstream of the grid. Shown in Fig. $1 \mathrm{c}$ is the solution that was implemented in F1: it consisted of a grid made of nonuniformly spaced rods, which were placed upstream of the air inlet. The nonuniform grid solidity $\zeta(h)$ recreated an ABL velocity profile in $\mathrm{F} 1$, and the wake of the rods seeded the flow with turbulent eddies, which mimicked the high-frequency part of the ABL turbulence spectrum that was of interest in this test. In addition, a variant of the grid with uniformly spaced rods was found to produce turbulence while keeping the mean flow uniform.

The design of the ABL grid is described in Sec. II. The preliminary grid testing and characterization in a small-sized wind tunnel are described in Sec. III. The final acceptance and characterization of the grid in the larger $\overline{\mathrm{F} 1}$ wind tunnel are described in Sec. IV. An insight into the effect of the two grid configurations on air inlet performance is contained in Sec. $\underline{\text { V. }}$.

\section{Theoretical Design of the ABL Grid}

The ABL characteristics have been studied for the mean flow (see Sec. II.A) and for the turbulent fluctuations (see Sec. II.B) to set up targets regarding the boundary-layer flow that need to be reproduced in the tunnel. Then, a theoretical and empirical basis for predicting the flow downstream of the grid was formulated again for the mean flow (see Sec. II.C), and then for the turbulent fluctuations (see Sec. II.D). These elements together have led to the definition of an optimized grid configuration.

\section{A. Mean Velocity Distribution in the ABL}

To set the design targets, the ABL characteristics for mean and fluctuating flows were taken from Refs. [17-19]. These sources propose to model the velocity distribution $U(\bar{h}) \overline{\text { using the following }}$ logarithmic law parameterized by the roughness height $h_{R}$ and a wind velocity at a height of $10 \mathrm{~m} U_{10}$ :

$$
U(h)=U_{10} \frac{\ln \left(h / h_{R}\right)}{\ln \left(h_{10} / h_{R}\right)}
$$

The roughness height depends on the type of local terrain. For the application corresponding to the takeoff and landing phases of aircraft, the terrain to consider is that of an airport, which is mostly flat open terrain, presenting few obstacles to the wind. The reference (Ref. [19] p. 684) suggests using $h_{R} \approx 5 \mathrm{~cm}(0.15 \mathrm{ft})$, and this value was used in the grid design. To give an indication of the effect of such surface roughness on the wind velocity, the mean velocity at $4 \mathrm{~m}$ above ground (where the upper lip of a large underwing engine might be located) is $36 \%$ higher than at $1 \mathrm{~m}$ above the ground.

\section{B. Velocity Fluctuations in the ABL}

There exists a considerable number of turbulence models to represent the velocity fluctuations in the ABL (e.g., in Ref. [19], p. 695 quotes 25 different models). However, these models differ from one another only by the way they consider the large turbulent eddies, which are tens or even hundreds of meters across, in the lower atmosphere.

For our application, we were only interested in the eddies capable of interacting with the flow inside the air inlet, i.e., eddies smaller than the air inlet diameter $D$. Indeed, because of their size and time scale, larger eddies are perceived by the air inlet as a slowly varying uniform flow, and consequently the inlet response can be assumed to be quasi steady [20]. These slow large-scale flow variations are in fact simulated by varying the wind-tunnel conditions (velocity magnitude and direction relative to the inlet) during the test campaign. Therefore, large eddies do not need to be simulated, and the grid objectives were limited to properly reproducing only the high-frequency range of the turbulence spectrum, as introduced in Sec. I.

Looking at the length scales involved ( $D$ is of the order of a few meters on aircraft), this frequency range accounts for only a small part of the total turbulent kinetic energy (typically a few percent). Furthermore, this frequency range falls well inside the so-called universal range of the turbulent spectrum, where turbulence is isotropic and Taylor's hypothesis applies.

Following this reasoning, we chose the turbulence model proposed by von Kármán, which is used in both Refs. [18,19], to give the power spectral density $S_{u u}$ as a function of wavelength $k$ :

$$
\frac{k S_{u u}(k)}{\sigma_{u}^{2}}=\frac{4 k l_{u}}{\left(1+70.8\left(k l_{u}\right)^{2}\right)^{5 / 6}} \underset{k l_{u} \rightarrow+\infty}{\sim} \frac{0.115}{\left(k l_{u}\right)^{2 / 3}}
$$

This formula is plotted on Fig. 2. It depends upon only two parameters: 1) the kinetic energy of longitudinal fluctuations $\sigma_{u}^{2}$;

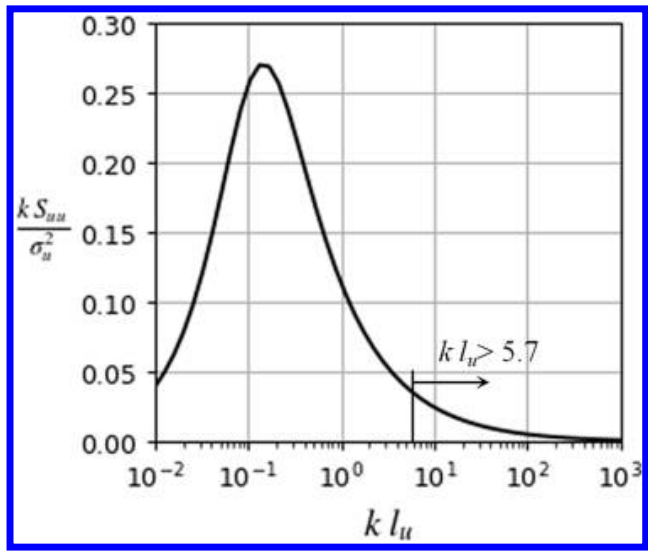

Fig. 2 Von Kármán turbulence energy spectrum. 
and 2) the length scale $l_{u}$, which is close to the turbulence integral length scale [18].

Numerical values of those two parameters according to our two sources $[\underline{18}, 19]$ are in good agreement. We ended up choosing as target values the data at $h=2.5 \mathrm{~m}$ from Ref. [18], which gave more details very close to the ground, as compared to Ref. [19], which covers the entire atmosphere, with some cutoffs below $10 \mathrm{ft}$ that set some parameters independent of height without any physical reasoning.

To illustrate the orders of magnitude involved, let us take as an example an inlet with a diameter of $D=2.5 \mathrm{~m}$ with the engine axis located at $h_{c}=2.5 \mathrm{~m}$ above the ground. At this height above ground, the turbulence ratio is $T_{u}\left(h_{c}\right)=\sigma_{u}\left(h_{c}\right) / U\left(h_{c}\right)=23.1 \%$. This is unusually high for external aerodynamic applications, in which typical turbulence ratio (e.g., in wind tunnels) are generally below $0.1 \%$. However, the length scale is $l_{u}=14.3 \mathrm{~m}=57 \times D$, which means that only the spectrum for $k l_{u} \geq 5.7$ is of interest in Fig. 2. For a local wind velocity of $U\left(h_{c}\right)=25 \mathrm{kt}$, this corresponds to a frequency larger than $5 \mathrm{~Hz}$, which is an order magnitude larger than required in Ref. [16]. From Eq. (2), this part of the spectrum only contains $5.3 \%$ of the total turbulent kinetic energy. Hence, the partial turbulence ratio

$$
\frac{1}{U} \sqrt{\int_{1 / D}^{+\infty} S_{u u}(k) \mathrm{d} k}
$$

is $\sqrt{0.053} \times 0.231=5.3 \%$. This was the order of magnitude of the partial turbulence ratio we established as a target for the grid design.

From this example, it is possible to perceive that the small-eddy range we are interested in is well described by the asymptotic behavior when $k l_{u} \gg 1$ [second part of Eq. (2)]. In this range, an increase in the turbulence energy $\sigma_{u}^{2}$ has the same effect as a reduction of the length scale $l_{u}$.

\section{Model for the Mean Flow Downstream of the ABL Grid}

The initial aerodynamic design of the grid was based on the theoretical approach described in Ref. [21], which was generalized to arbitrary velocity profiles in Ref. [22]. In this theory, the grid is modeled as a discontinuity of momentum as proposed by Batchelor [23]. When passing through the grid, 1) the flow experiences a pressure drop of $\Delta p=\frac{1}{2} \rho U^{2} K$, where $U$ is the local flow velocity and $K$ is the local resistance coefficient; and 2) the velocity component tangential to the grid is multiplied by a factor of $\alpha<1$, called the refraction (or deflection) coefficient.

The computations detailed in Ref. [1] allow one to predict the whole velocity field upstream and downstream of the grid, and they lead to the following relationship between the requested velocity distribution $U(h)$ downstream of the grid and the distribution of resistance coefficient $K(h)$ :

$$
K(h)=K_{0}-2\left(1+\frac{K_{0}}{1+\alpha_{0}}\right)\left(\frac{U(h)}{U_{Q}}-1\right)
$$

This equation is, however, established by considering only small disturbances to the tunnel upstream velocity: a linearizing hypothesis that was not well verified in the present work.

Two additional relationships between the grid solidity $\zeta$ and the grid resistance coefficient $K$ and refraction coefficient $\alpha_{0}$ are needed. We used the relationships proposed in Ref. [21] since more elaborate models did not significantly improve the results as found with other applications [느] as follows:

$$
\begin{aligned}
K & =\frac{\zeta}{(1-\zeta)^{2}} \\
\alpha_{0} & =\frac{1.1}{\sqrt{1+K_{0}}}
\end{aligned}
$$

Combining Eqs. (3-5) yields a second-order polynomial equation in the grid solidity $\zeta(h)$ to be solved for each height $h$ considered. The mean resistance coefficient of the grid $K_{0}$ is arbitrary, which means that several grids of different mean resistance can lead to the same velocity distributions, provided the solution $K(h)$ remains positive everywhere (since the grid is a passive device that can only dissipate energy from the flow). The rod diameter $d$ can also be freely chosen since a given solidity can be achieved with rods of different diameters by changing their spacing. Those two free parameters are the only ones available for optimizing the turbulent content using considerations from Sec. II.D.

This assessment leads to a continuous distribution of grid solidity $\zeta(h)$ that equates to the position of a discrete number of grid rods, thanks to an iterative algorithm starting at the floor and outlined in Fig. 3. This yields the number and the location of the rods in the grid as reported in Table $\underline{1}$.

\section{Model for the Turbulent Fluctuations Downstream of the ABL Grid}

The rods produce turbulence in their wakes. This turbulence is convected downstream, where it progressively becomes homogeneous and isotropic, and is progressively dissipated [25]. The main characteristics of the turbulence downstream of the grid were predicted from a literature survey [25-28] to set up an empirical model that could be used in the grid design.

As a first step, we modeled the amount of turbulence kinetic energy produced by the grid as a function of grid solidity. Measurements reported in Ref. [27] downstream of uniform grids with varying solidity at a distance of 38 times the rod spacing $M$ were used for this purpose. A polynomial regression of their measurements for $0.1 \leq \zeta \leq 0.5$ was derived:

$$
{\frac{\sigma_{u}}{U\left(\frac{x}{M}=38\right)}}=\left(1.179+4.536 \zeta-12.50 \zeta^{2}+50.00 \zeta^{3}\right) \times 0.01
$$

As a second step, we modeled the rate of decay of this turbulence energy downstream of the grid. In Ref. [26], the author proposes to model this decay by a power of the downstream distance $x$ divided by the rod spacing $M$, for distances larger than $(x / M)>30$ :

$$
\frac{\sigma_{u}}{U}={\frac{\sigma_{u}}{U}}_{\left(\frac{x}{M}=38\right)}\left(\frac{x / M}{38}\right)^{-0.6}
$$

Figure 4 compares the results obtained by various authors $[25-$ 28]. These authors used grids of different solidities ( 0.15 to $0 . \overline{29})$. To make their results more comparable, they were extrapolated to a common solidity of 0.26 using Eq. (6). Like the authors from Ref. [28], for an undetermined reason, we measured during the physical grid characterization (see Sec. III.B) larger turbulent kinetic energy downstream of the grid than the other references. Therefore, in our model, by predicting the turbulence ratio downstream of the grid, the result of Eq. (6) was multiplied by an empirical factor of 1.45 .

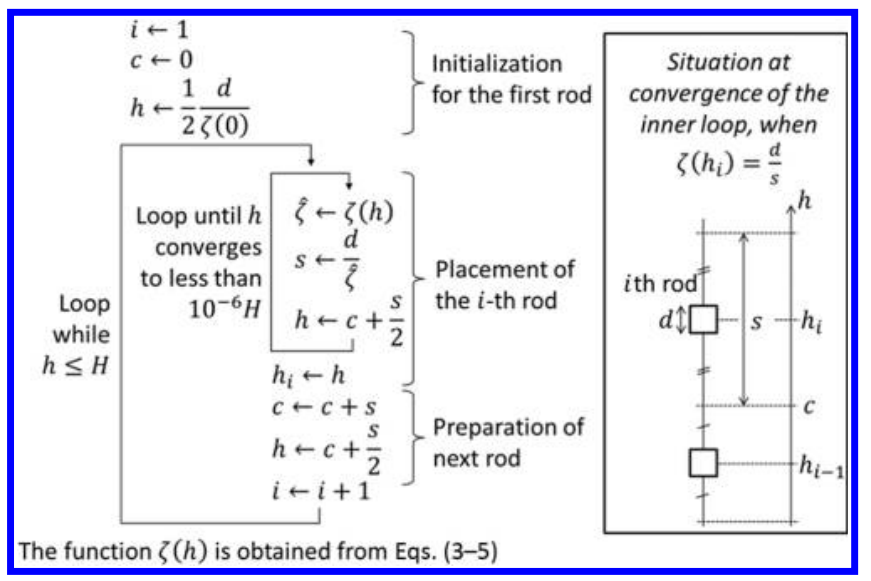

Fig. 3 Iterative algorithm for the positioning of the rods. 
Table 1 Placement of

the rods of the grid tested in

Sec. III

$\left(h_{R} / H=3 . \overline{7} \times 10^{-3}\right.$

$d / H=0.222$, and

$\left.K_{0}=0.78\right)$

\begin{tabular}{lc}
\hline \hline Rod number $i$ & Height, $h_{i} / H$ \\
\hline 1 & 0.0211 \\
2 & 0.0650 \\
3 & 0.1128 \\
4 & 0.1639 \\
5 & 0.2189 \\
6 & 0.2767 \\
7 & 0.3378 \\
8 & 0.4028 \\
9 & 0.4722 \\
10 & 0.5467 \\
11 & 0.6278 \\
12 & 0.7161 \\
13 & 0.8150 \\
14 & 0.9300 \\
\hline \hline
\end{tabular}

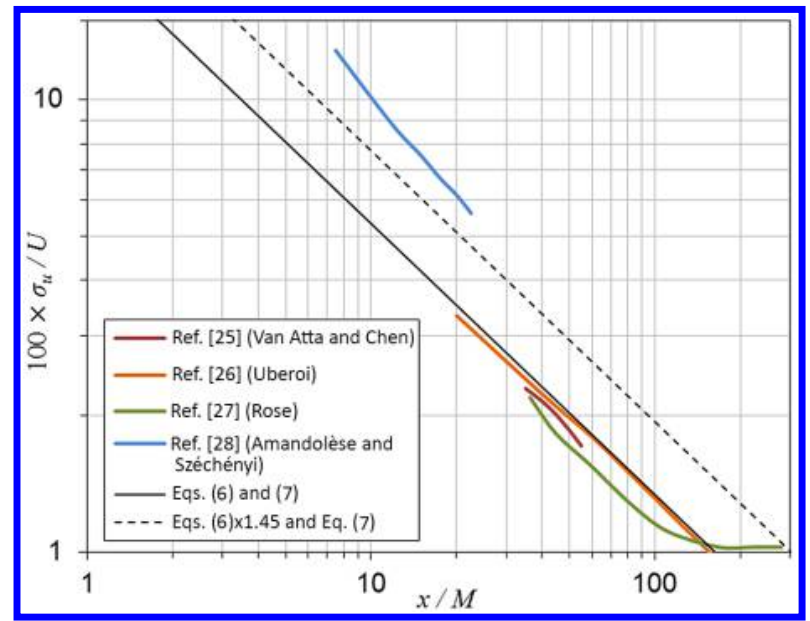

Fig. 4 Decay of turbulence downstream of a grid of 0.26 solidity

As a third step, it was necessary to model how this turbulent kinetic energy is spectrally distributed. Examination of results reported in Ref. [25] reveals that the integral length scale $l_{u}$ downstream of the grid is about half the rod spacing $M$, and it only slowly increases with downstream distance, as verified in Ref. [26]. Consequently, we simply retained for our model the following value to be used in the von Kármán spectrum Eq. (2):

$$
l_{u}=\frac{1}{2} M
$$

The whole modeling assumes that the aforementioned relationships [Eqs. (2, 6-8)], established on different grids of uniform solidity, remain locally valid downstream of a single grid of varying solidity.

\section{E. Scaling Considerations}

The scaling of this situation requires additional nondimensional parameters compared to usual wind-tunnel testing since the experiment should replicate 1) the nondimensional ground distance $h_{c} / D$; 2) the Mach and Reynolds numbers based on the reference atmospheric velocity $U_{10}$ and inlet diameter $\left.D ; 3\right)$ the nondimensional velocity distribution $\frac{U}{U_{10}}(h / D)$ in the ABL; 4) the turbulence ratio distribution $T_{u}(h / D)$ in the ABL; and 5) the nondimensional turbulence length scale distribution $\frac{l_{u}}{D}(h / D)$ in the ABL.

Let us take again the example of an air inlet of diameter of $2.5 \mathrm{~m}$ with the engine axis located at $2.5 \mathrm{~m}$ above ground. In F1, due to the rig size and characteristics, the inlet model needs to have a diameter of about $40 \mathrm{~cm}$, i.e., a scaling factor of about $\lambda=1 / 6$. If the reference wind velocity $U_{10}$ is given at $h_{10}=10 \mathrm{~m}$, the same velocity should be achieved in the tunnel at a height of $\lambda h_{10}=1.67 \mathrm{~m}$ above the ground panel. (To be rigorous, we should be using the Mach number here; but since the tunnel temperature is ambient, this is equivalent to velocity.) At the height of the air inlet, the full-scale turbulent length scale is $l_{u}=14.3 \mathrm{~m}$. In the tunnel, a turbulent length scale of $\lambda l_{u}=2.4 \mathrm{~m}$ at the height of the air inlet model of $\lambda \times 2.5=0.4 \mathrm{~m}$ is requested. In that case, since the tunnel can operate up to 3.85 bar, the model Reynolds number will be $64 \%$ of the full-scale Reynolds number $(\lambda \times 3.85$ bar $/ 1$ bar $=0.64)$. With the turbulence grid, it is not possible to fully represent the distribution of the turbulence ratio and turbulence length scale, even if the tendencies are the same (increase of $T_{u}$ and decrease of $l_{u}$ when getting closer to the ground).

Regarding scaling, we also choose to use rods of nearly square cross sections instead of the more classical circular cross sections, with the hope that the grid flow should be less sensitive to changes in Reynolds number. This is a desirable characteristic since the grid was operated over a wide range of velocities and densities in the F1 pressurized wind tunnel.

\section{Proof of Concept and Turbulence Measurements}

\section{A. F2 Wind-Tunnel Setup}

The F2 wind tunnel is a low-speed low-turbulence atmospheric wind tunnel [29]. It features a modular test section (1.4 m wide $\times 1.8 \mathrm{~m}$ high) that can be equipped with large transparent wall panels, and it is surrounded by a three-degree-of-freedom traverse system. This makes the F2 particularly well suited to the use of optical measurement systems. It was for this reason that the F2 was used for the development of the grid and the characterization of the turbulence it generated.

ABL grid prototypes were tested inside the tunnel using off-the-shelf Bosch ${ }^{\mathrm{TM}} 40 \times 40 \mathrm{~L}$ aluminum rods with a square cross section of size $40 \times 40 \mathrm{~mm}(d=4 \mathrm{~cm})$ with rounded edges. The grid was spread over the entire height $(H=1.8 \mathrm{~m})$ of the test section entrance (see Fig. 5). With this geometry, the grid was approximately a $4 / 5$-scale prototype of the grid tested in the F1 wind tunnel. The tunnel velocity $U_{A}$ was measured using a Prandtl antenna on the tunnel ceiling $3.55 \mathrm{~m}(2 \times H)$ downstream of the grid. The flow was seeded with atomized olive oil, and velocity measurements (mean and fluctuations) were carried out with an existing three-component laser Doppler velocimetry (LDV) system. An average acquisition rate of $10 \mathrm{kHz}$ was achieved, and spectral distributions were determined from 50,000 samples. Taylor's hypothesis was used to transform spectral distribution in the time frequency domain into distribution in spatial frequency. All measurements presented in this paper were taken as $3.55 \mathrm{~m}(2 \times H)$ downstream of the grid, which roughly corresponded to the position of the air inlet model installed in the F1 wind tunnel. A freestream velocity of $U_{A}=40 \mathrm{~m} / \mathrm{s}$ was used throughout the testing.

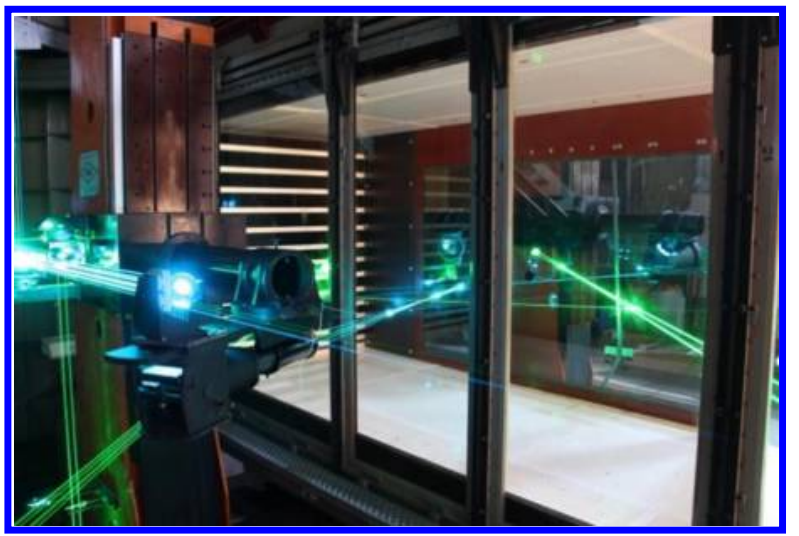

Fig. 5 Prototype grid installed in the F2 wind tunnel during LDV measurements. 


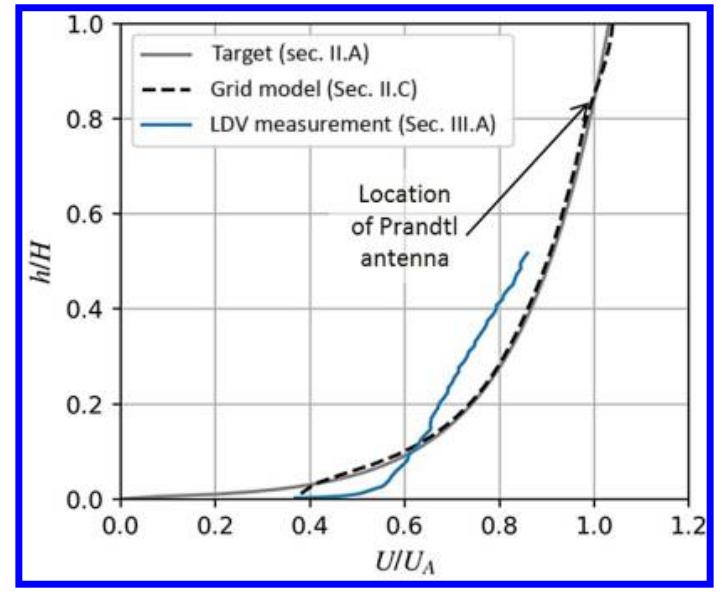

Fig. 6 Mean axial velocity downstream of the grid.

\section{B. Main Results for the Mean Flow}

Concerning the mean flow distribution, the F2 test revealed the limits of the theoretical model presented in Sec. II.C, as can be seen on Fig. 6. Close to the floor, the grid (created using the theoretical model) exhibited insufficient resistance to the flow; hence, too large a velocity existed. This in turn distorted the middle part of the flow distribution. Empirical variants of the grid were then tested using a trial-and-error technique to improve the velocity distribution. It was also interesting to find that the individual grid wakes had completely dissipated at the simulated inlet model location.

The pressure distribution on the test section walls displayed in Fig. 7 shows a close agreement of the model compared with experimental data. Interestingly, as predicted by the grid modeling, the flow is fully established at $(x / H) \approx 1$ downstream of the grid.

\section{Main Results for the Turbulent Fluctuations}

Concerning the velocity fluctuations, the turbulence ratios $T_{u}, T_{v}$, and $T_{w}$ plotted on Fig. 8 are about $5 \%$ over most of the tunnel height and rise to more than $10 \%$ very close to the floor. The turbulence is fully isotropic (except in the last $20 \mathrm{~cm}$ close to the floor, where vertical velocity fluctuations are damped by the presence of the wall, as found in the ABL). The grid model from Sec. II.D approximately replicates this behavior. Of course, the target curve is the partial turbulence ratio for small eddies only.

The spectral distribution shown in Fig. 9 is compared to the actual ABL spectrum (scaled according to Sec. II.E) and to the model of Sec. II.D. In this figure, the spatial frequency $k$ is multiplied by the rod diameter $(d=4 \mathrm{~cm})$ to form a Strouhal number. Due to experimental limitations, these measurements were obtained on a grid that was not identical to the ones of the data of Figs. 6 and 8 but very similar, and they presented about the same vertical distribution of turbulence intensity.

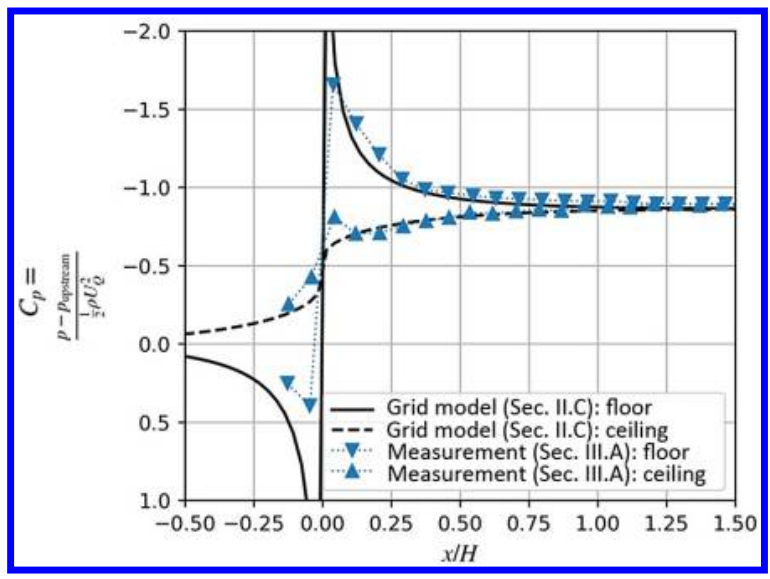

Fig. 7 Pressure distribution on the F2 test section walls.

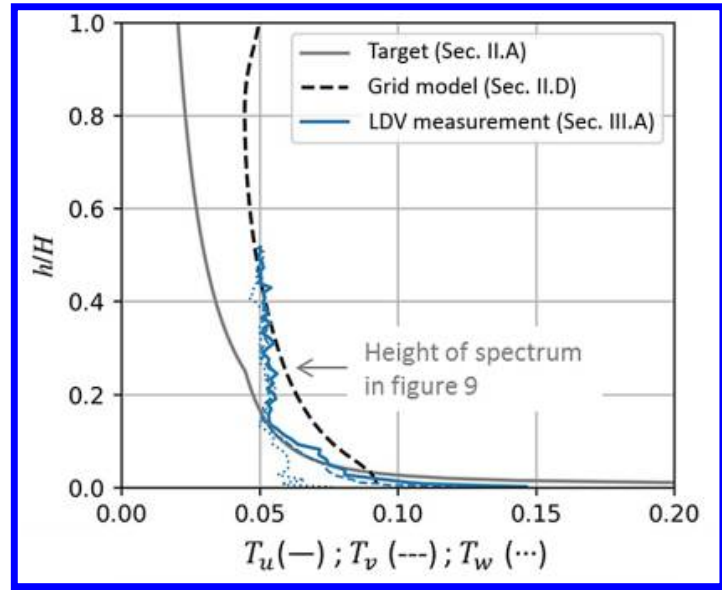

Fig. 8 Turbulence ratio downstream of the grid.

The small-eddy range of $k D \geq 1$ that we are trying to replicate corresponds to $k d \gtrsim d / D=0.13$, since in F1 the chosen rod diameter was $d=5 \mathrm{~cm}$, and the inlet diameter was imposed by the test setup as $D \approx 40 \mathrm{~cm}$ (see Sec. II.E). Consequently, looking at the spectral distribution, about $40 \%$ of the grid turbulence kinetic energy falls in the small-eddy range $k D \geq 1$. Since the grid total turbulence ratio $T_{u}$ is about $5 \%$, at a height of the order of $D$ above the ground, this corresponds to a partial turbulence ratio of $\sqrt{0.4} \times 5 \%=3.2 \%$. This fell slightly short of the expected $5.3 \%$, which was calculated as a target at the end of Sec. II.B, but this was still a good order of magnitude and a considerable amount of turbulence compared to the normal laminar flow of the wind tunnel. One can also note from Fig. 9 that the spectral distribution of this turbulence energy downstream of the grid was close to that of the ABL over the frequency range of interest.

\section{Grid Manufacture and Validation Tests in the F1 Wind Tunnel}

\section{A. Grid Geometric Layout}

The F1 test section is $4.5 \mathrm{~m}$ wide, $3.5 \mathrm{~m}$ high, and $8 \mathrm{~m}$ long. To simulate an air inlet in crossflow, a flat dummy floor panel of $6.2 \mathrm{~m}$ in length was placed in the test section to simulate the ground; see Figs. 1 and 10 .

The grid was made of off-the-shelf Bosch $50 \times 50 \mathrm{~L}$ aluminum rods with a square cross section of $50 \times 50 \mathrm{~mm}$ in size and rounded edges. The rods span the entire width of the test section entrance and are spread over the entire height of the wind-tunnel test section, as shown in Fig. 10. In this figure, note that the whole setup is rotated by 90 deg compared to the real-life situation; therefore, the direction of the height $h$ is horizontal. The rods were supported by two tracks: one

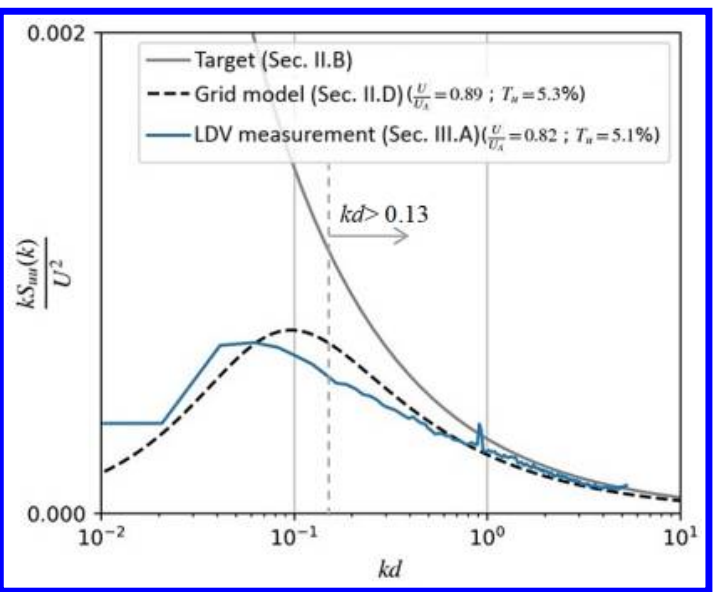

Fig. 9 Spectrum of $u$ fluctuations downstream of the grid and $0.26 \times H$ $(0.47 \mathrm{~m})$ above the ground. 


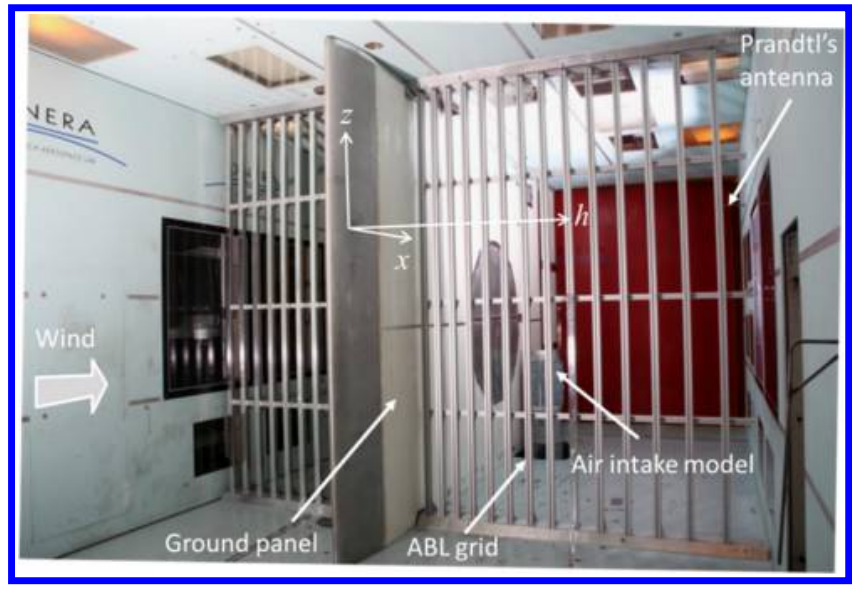

Fig. 10 F1 test section configured for air inlet testing with the ground panel and the ABL grid. The air intake model is described in Sec. $\underline{\text { V.A. }}$

on the floor of the tunnel, and one on the ceiling. Rod position could be freely adjusted along the tracks, as required. This allowed the rods to be moved in association with the ground panel when simulating different inlet ground heights. This also allowed different rod distributions to be set, e.g., to generate different velocity profiles. The lower side of the ground panel was fitted with a homogeneous grid, with a resistance coefficient equal to the average resistance coefficient of the ABL grid $K_{0}$, in order to balance the mass flow on each side of the ground panel.

Obviously, the grid considerably increases the loss coefficient of the tunnel (loss in total pressure around the circuit divided by the dynamic pressure in the test section), which rose from about 0.3 with the empty test section [8] to about one with the grid installed. The F1 tunnel fan coped with this additional flow resistance without approaching surge, thanks to its variable blade pitch capability.

During the first runs, significant aeroelastic vibrations of the rods were encountered. Those vibrations were attributed to a combination of vortex shedding and galloping, and they were suppressed by reinforcing the grid with three transverse rods as used in Ref. [30] (the horizontal rods that can be seen on Fig. 10). Similar problems and solutions were also reported with the spires used in Ref. [16]. The operating range of the grid was extended to $30 \mathrm{kt}$ at the air inlet height at maximum tunnel pressure (3.85 atmospheres).

\section{B. Grid Validation Tests}

The validation test relied on particle image velocimetry (PIV) measurements of the mean flow downstream of the grid, focusing on the location where air inlet models are mounted. Three-component PIV measurements were carried out using a transverse laser sheet and two 4 megapixel cameras operated at five images per second. More details on the standard PIV hardware in F1 can be found in Refs. [31,32]. During this test, a large field of view was used, which reduced the accuracy of velocity measurements to an estimated $5 \%$ of $U_{A}$.

Thanks to the preliminary test in the F2 wind tunnel (Sec. III), only one trial-and-error adjustment of the rod height distribution was necessary in F1 to obtain the results plotted in Fig. 11. The distribution of the mean axial velocity $U$ closely followed (within $\pm 10 \%$ ) that of the ABL profile of Eq. (1), with $h_{R}=2.2 \mathrm{~cm}$ (at wind-tunnel scale); and the mean transverse velocities $V$ and $W$ remained small.

Using the example of Sec. II.E with a scale factor of 1:6, the fullscale roughness parameter being simulated was about $13 \mathrm{~cm}$. This was larger than the objective of $5 \mathrm{~cm}$ described in Sec. II.A, but this was still very representative of the flat open terrain we were trying to replicate in the wind tunnel.

Unfortunately, LDV measurements were not possible in the F1 wind tunnel, and PIV was unable to provide reliable measurements of the turbulent velocity fluctuations. Therefore, the best available characterization of the turbulence in $\mathrm{F} 1$ had to be based on the measurements downstream of the scaled-down grid in the F2 wind tunnel, presented in Sec. III.B.

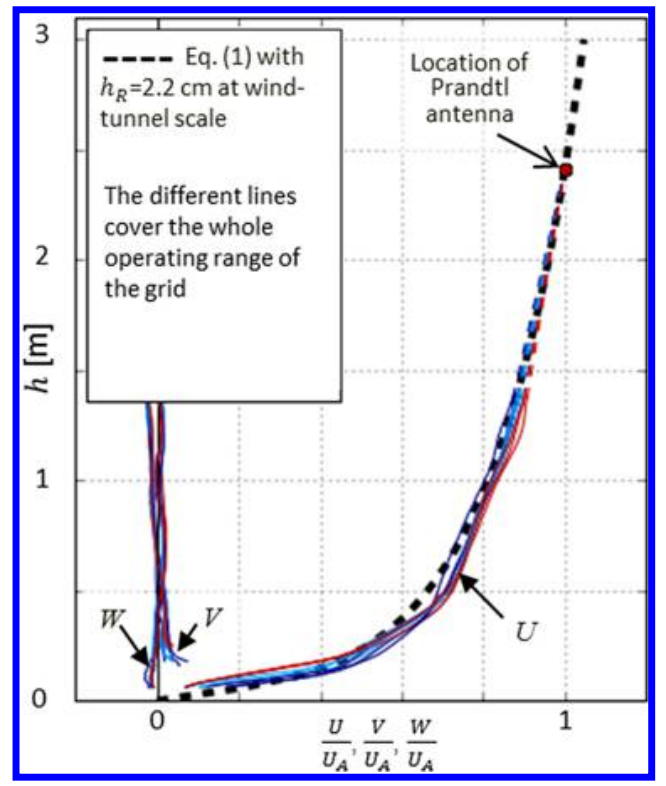

Fig. 11 Spanwise-averaged PIV measurements of the flow velocity around the air inlet location.

Wind-tunnel users were interested in separating the effect of the gradient in mean velocity from the effect of the turbulence of the flow. For this reason, a variant of the grid with uniform rod spacing was also made available simply by rearranging the rods in their ground and floor tracks. The uniform grid is designed to produce only turbulence and no gradient in the mean flow velocity.

\section{Effect of the ABL Grid on Air Inlet Performance}

\section{A. Test Setup}

The effect of the grid on air intake aerodynamic performance was performed using a model named "EPICE 2." This inlet was representative of modern short-cowl air intakes of about $1 / 6.5$ scale, with a fan plane diameter of $338 \mathrm{~mm}$. The ground panel was installed $237 \mathrm{~mm}$ from the fan plane axis. This model was tested in crosswind (with a sideslip angle of $90 \mathrm{deg}$ ) and with a wind velocity from 25 to $35 \mathrm{kt}$ (13 to $18 \mathrm{~m} / \mathrm{s}$ ). The results at $25 \mathrm{kt}$ are only presented in this paper because the results at higher wind velocities were qualitatively the same, with a smaller fan operating range and a smaller hysteresis loop. All experiments were carried out with the tunnel pressurized at 3.8 bar, resulting in a Reynolds number of about $60 \%$ of full scale. The inlet was equipped with 104 total pressure taps in the fan plane

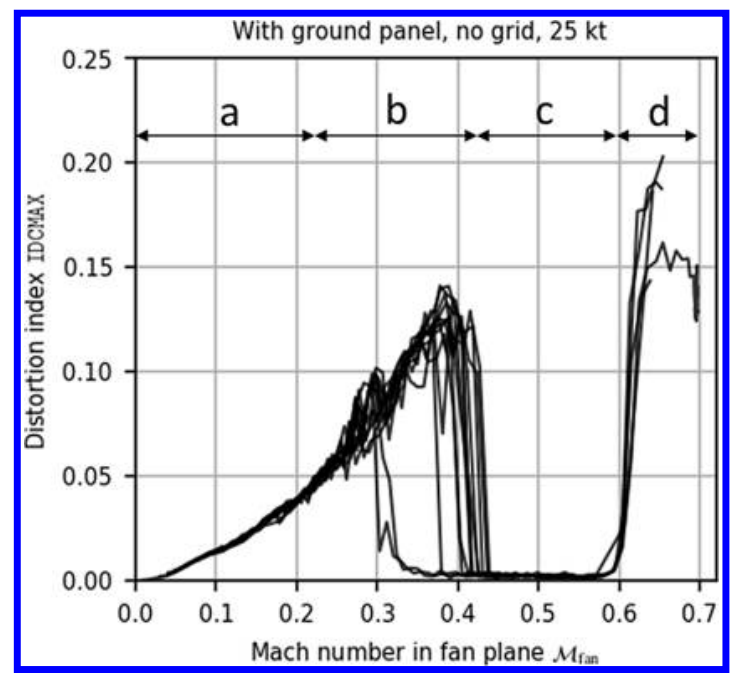

Fig. 12 Distortion index without ABL grid over 10 successive data acquisitions (IDCMAX = maximum circumferential distortion index of the flow in the fan plane of the air inlet). 


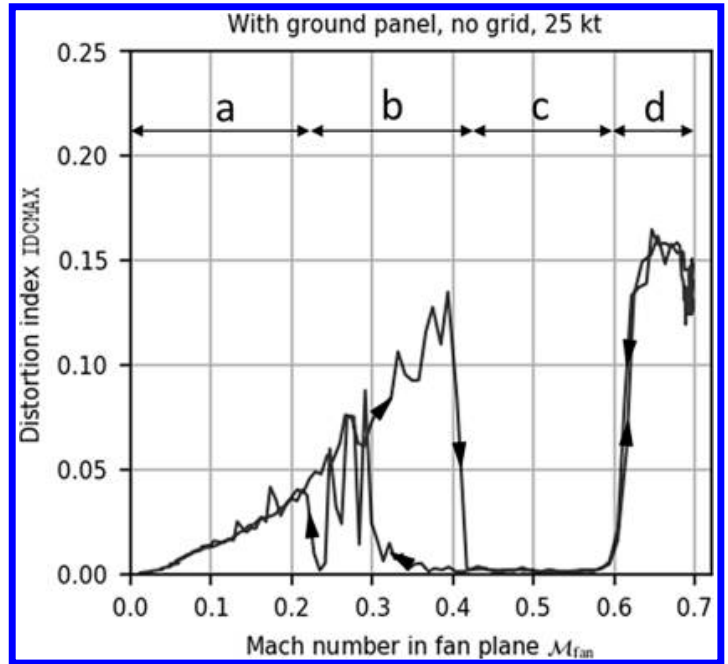

Fig. 13 Inlet distortion index without ABL grid installed showing a hysteresis loop.

distributed over eight rakes. The total pressure data were used to compute the maximum circumferential distortion index IDCMAX [2]. Unfortunately, the rake at $150 \mathrm{deg}$ in the inlet model broke due to fatigue loadings experienced during the experiment. Consequently, only data from the remaining seven rakes (96 taps) were used to compute the distortion indices. The mass flow entering the air inlet was measured using Venturi mass flow control devices.

\section{B. Air Inlet Flow Without the ABL Grid Installed}

Without the grid installed, the distortion index with increasing inlet mass flow and at a fixed wind-tunnel velocity of $25 \mathrm{kt}$ behaved as depicted in Fig. 12. This figure contains 10 curves corresponding to 10 successive measurements carried out within $1 \mathrm{~h}$ of each other, under identical conditions, and during the same wind-tunnel run. In this figure and the following ones, the mass flow is translated into a mean Mach number at the fan plane $\mathcal{M}_{\text {fan }}$ using a one-dimensional isentropic flow formula. The accuracy of the mass flow, pressure, and temperature measurements was sufficient to ensure the accuracy of
$\mathcal{M}_{\text {fan }}$ was always better than 0.01 . At the low mass flow (regions a and b in Fig. 13), the flow entering the inlet separated on the windward lip and generated an area of low total pressure in the left region of the fan plane (Figs. 14a and 14b). As the mass flow increased, this separated area slowly developed in size and shape, until it suddenly disappeared as $\mathcal{M}_{\text {fan }}=0.45$ was approached (see Fig. $14 \mathrm{c}$ ). With further increase in mass flow, a supersonic area grew on the windward lip. When the shock wave in this area became too intense, it caused the boundary layer on the lip to separate again (see Fig. $14 \mathrm{~d}$ ). At $\mathcal{M}_{\text {fan }}=0.7$, the flow downstream of the fan plane became choked. So, this mass flow was an upper bound for the inlet mass flow. Region c represents the engine operating range, in which the flow was attached and the total pressure in the fan plane was uniform, apart from the ground vortex that sometimes impinged on the rake at $150 \mathrm{deg}$ (Fig. 14c).

It is easily seen on Fig. 12 that the reattachment mass flow exhibited significant scatter in region $\mathrm{b}$. In this region, the detached state of the flow around the windward lip appeared to be metastable: the flow was transitioning spontaneously from a detached to an attached state, but only after a random duration that was very long with regard to the other time scales of the flow. This phenomenon was similar to the bistability one reported in Ref. [33]. During experiments performed at a fixed mass flow with this model (at $25 \mathrm{kt}$ crosswind and $\mathcal{M}_{\text {fan }}$ as low as 0.25 ), a spontaneous reattachement was observed after more than $40 \mathrm{~s}$ of nonevolving flow conditions in the metastable region (region b). Since this time scale was also large with respect to the duration of the mass flow sweep during the experiment, the probability of observing reattachment when traversing the metastable region was well below one. This explains the scatter between the different runs shown in Fig. 12.

Interestingly, the metastable region (region b) approximately coincides with the hysteresis region, as shown on Fig. 13. In this figure, the mass flow in the air inlet was slowly increased up to choking, and then it was slowly decreased down to zero. The plot shows a hysteresis loop in mass flow already discussed in Refs. [4, 5 ], and it is similar to the hysteresis in angle of attack reported in Refs. [9,34].

\section{Air Inlet Flow with the Uniform Grid Installed}

Figure 15 displays results obtained with a uniform turbulence grid. With this test setup, data scatter associated with metastability almost completely disappeared, as well as the hysteresis loop; i.e., there was

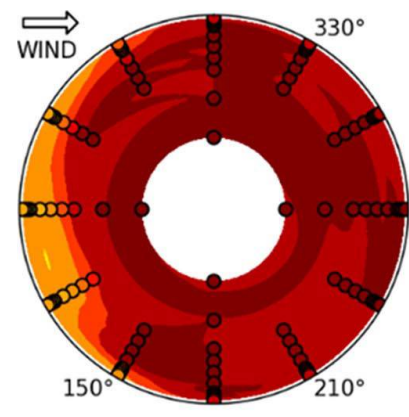

a) $\mathcal{M}_{\text {fan }}=\mathbf{0 . 2 0}$

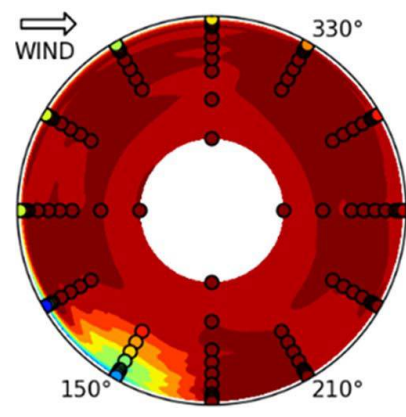

c) $\mathcal{M}_{\text {fan }}=\mathbf{0 . 5 5}$

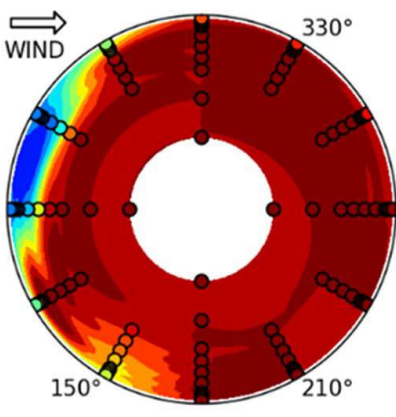

b) $\mathcal{M}_{\text {fan }}=\mathbf{0 . 3 5}$

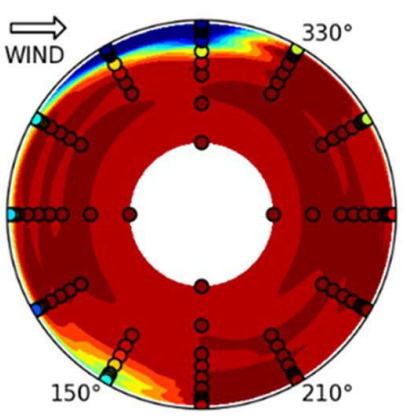

d) $\mathcal{M}_{\text {fan }}=\mathbf{0 . 6 5}$

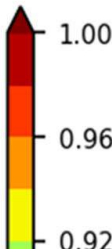

0.92

$\frac{p_{i}}{P_{i 0}}$

0.84

0.80

Fig. 14 Distribution of total pressure at the fan plane without the ABL grid installed. 


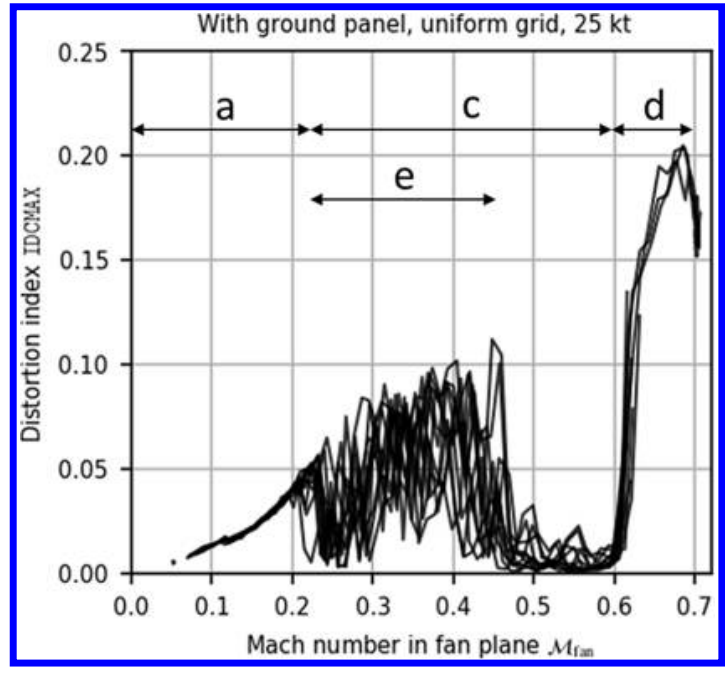

Fig. 15 Inlet distortion index with a homogeneous grid installed over 10 successive data acquisitions.

no longer a region $b$ in Fig. 15. The spikes in the distortion index in the region labelled region e in Fig. 15 are not due to flow separation but to the ground vortex now impinging the rake at $180 \mathrm{deg}$, as can be seen from Fig. 16b.

\section{Air Inlet Flow with the ABL Grid Installed}

With the ABL grid installed, the F1 wind tunnel was run to achieve an upstream velocity of $25 \mathrm{kt}$ at the center of the air inlet, based on data described in Sec. IV.B. Using the velocity profile in Fig. 11, this corresponds to a velocity of about $50 \mathrm{kt}$ at the Prandtl antenna in the test section.

Figure 17 shows results obtained with the turbulence grid in the ABL configuration of Sec. IV.B. Again, the hysteresis loop and the scatter associated with the metastability almost completely disappeared. However, the performance of the air inlet was dramatically reduced: the lip flow never reattaches and region c disappears from Fig. 17, as was verified in Fig. 18 (note the absence of the data from the rake arm at $150 \mathrm{deg}$ ).

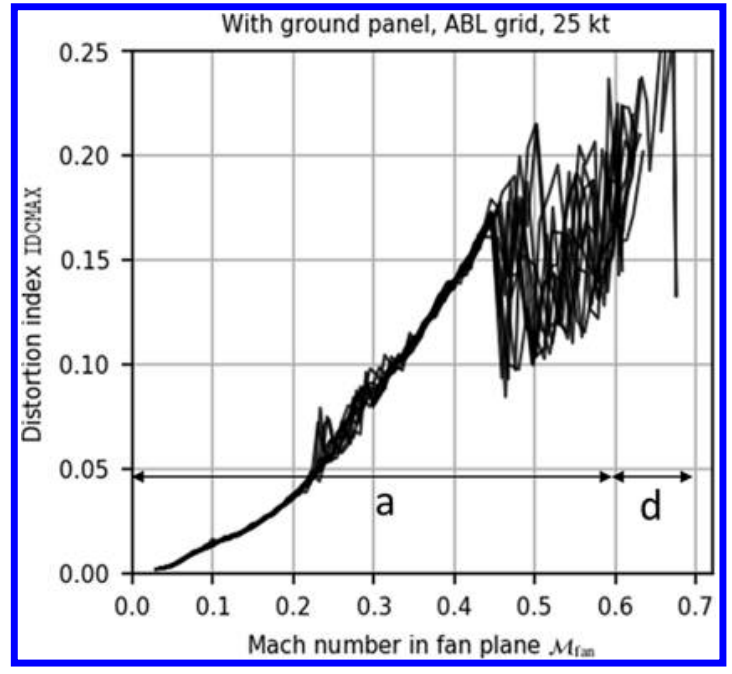

Fig. 17 Inlet distortion index with the ABL grid installed over 10 successive data acquisitions.

\section{E. Discussion}

We believe that the effects of the turbulence grids on the air inlet performance may be for the following reasons:

1) The level of turbulent kinetic energy, even limited to the small eddies as explained before, is thought to be sufficient to force transition to turbulence of any laminar boundary layer developing on the inlet lip, therefore modifying the boundary layer's characteristics and its resistance to separation, as exemplified in Ref. [4].

2) The metastability phenomenon is suppressed by the grid, considering the increased energy available to perturb the flow and trigger transition from detached to attached states.

3) The ground vortex is interacting with the turbulence field. More specifically, it may wander around its mean position and interact with the flow separation on the inlet lip. This phenomenon might also explain the failure of the $150 \mathrm{deg}$ rake arm that was frequently impacted by the vortex.

4) For the ABL grid, the nonuniformity of the mean velocity profile causes significant variation of local crossflow velocity near the inlet,

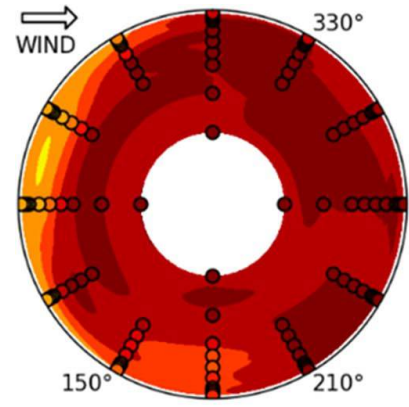

a) $\mathcal{M}_{\text {fan }}=\mathbf{0 . 2 0}$

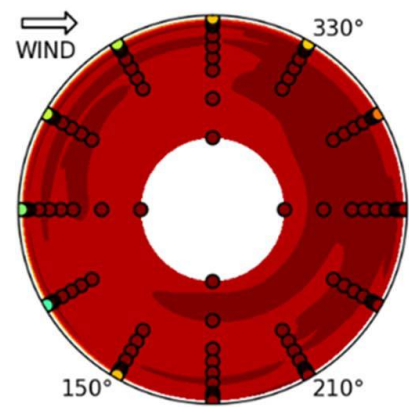

c) $\mathcal{M}_{\text {fan }}=\mathbf{0 . 5 5}$

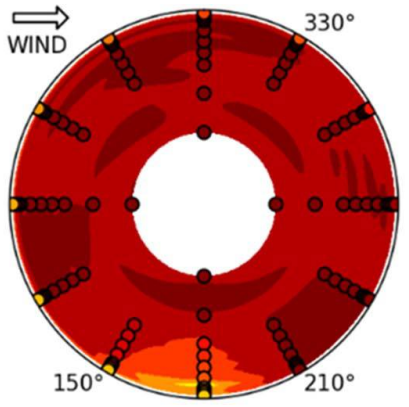

b) $\mathcal{M}_{\text {fan }}=\mathbf{0 . 3 5}$

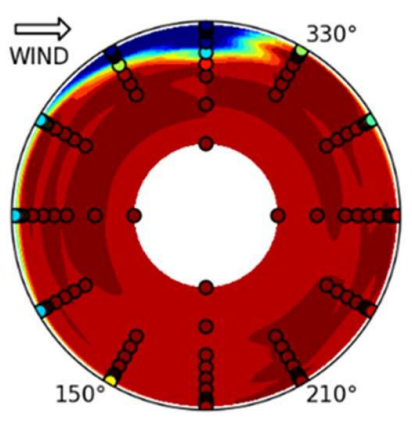

d) $\mathcal{M}_{\text {fan }}=\mathbf{0 . 6 5}$
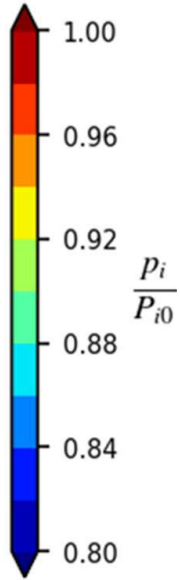

Fig. 16 Distribution of total pressure at the fan plane with a homogenous grid installed. 


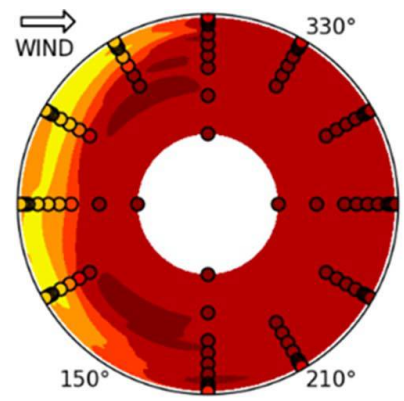

a) $\mathcal{M}_{\text {fan }}=\mathbf{0 . 2 0}$

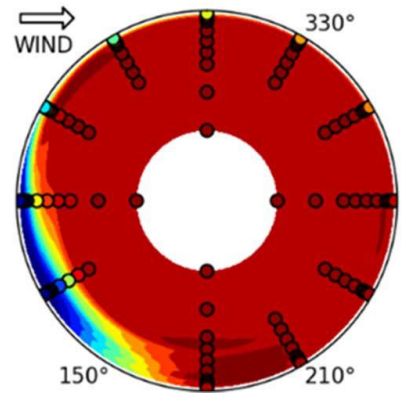

c) $\mathcal{M}_{\text {fan }}=\mathbf{0 . 5 5}$

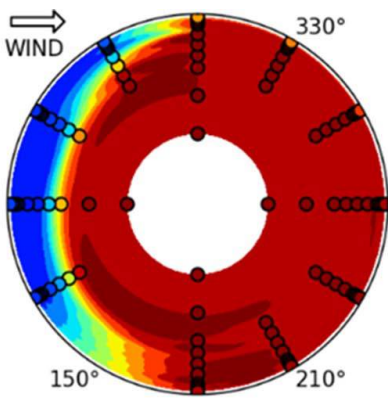

b) $\mathcal{M}_{\text {fan }}=\mathbf{0 . 3 5}$

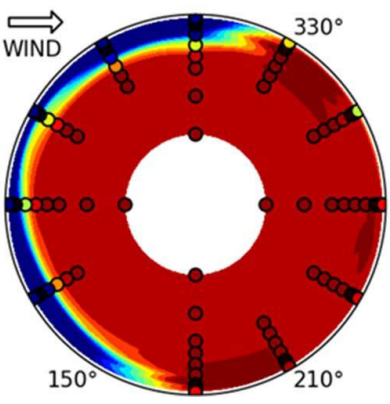

d) $\mathcal{M}_{\text {fan }}=\mathbf{0 . 6 5}$

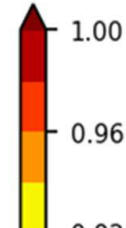

0.92

$\frac{p_{i}}{P_{i 0}}$

0.88

0.84

Fig. 18 Distribution of total pressure at the fan plane with the ABL grid installed.

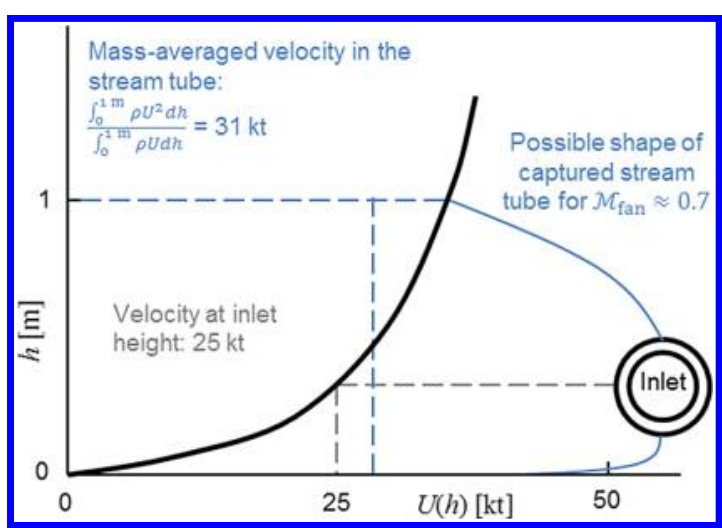

Fig. 19 Velocity averaging across the stream tube height.

therefore impacting the mass flow distribution in the captured stream tube. Hence, the velocity field in and around the inlet is nonuniform, and the occurrence of flow separations is affected.

Concerning this last point, one has to underline that the velocity averaged across the captured stream tube (as proposed in Refs. [7,20]) is likely to be higher than the empty test section velocity at the height of the air inlet: even more if the average is mass flow weighted and not simply area weighted, as illustrated in Fig. 19.

Finally, using a nonuniform velocity profile raises the question about the proper crossflow velocity to generate upstream of the inlet. With the example of Sec. II.E, imposing $25 \mathrm{kt}$ at $2.5 \mathrm{~m}$ above the ground means $33 \mathrm{kt}$ at $10 \mathrm{~m}$ above ground in the $\mathrm{ABL}$, which is the height typically used for meteorological measurements of the wind. In this situation, it is unclear to the author if the air inlet experiment is demonstrating the ability of the aircraft to cope with a $25 \mathrm{kt}$ crosswind or a $33 \mathrm{kt}$ crosswind in terms of certification. This is a question for the certifying authorities, as well as the inlet designers.

\section{Conclusions}

A turbulence grid to simulate the relevant features of the mean and turbulent flows in the atmospheric boundary layer was designed for use in the ONERA F1 wind tunnel. This was done especially for crosswind air inlet testing. Theoretical models have been used to support the design and the interpretation of results. A test with a prototype grid in the smaller ONERA F2 wind tunnel has helped validate the concept and correct the design and measurement of the grid turbulence. The final grid design was then manufactured and successfully tested in the $\mathrm{F} 1$, up to stagnation pressures of 3.85 atmospheres at $30 \mathrm{kt}$.

The effects of two different grids were investigated on a typical air intake model in $90 \mathrm{deg}$ crossflow. The uniform grid generated a higher level of turbulence in the wind tunnel, which was found to significantly enlarge the operating range of the intake. The ABL grid, which also changed the velocity distribution in the wind tunnel, was found to have an opposite effect, suppressing reattachment on the inlet lip. However, the proper upstream velocity to consider for the comparison between the two grid configurations is unclear. Both grids suppressed most of the hysteresis and metastability phenomena that were observed on the air intake, with no grid installed.

The ABL grid is now available for customers and researchers testing in the $\mathrm{F} 1$ wind tunnel to represent the engine inlet in crossflow in ground effect. This new device is expected to help improve the prediction of air inlet performance in crossflow and ground effect situations, which are topics that still attracts significant experimental attention [35].

\section{Acknowledgments}

This research was funded by ONERA's research budget as part of the continual improvement of its wind-tunnel testing services. The author would like to gratefully acknowledge the role of Test Engineers Hyacinthe Buxadera, Sylvain Massebœuf, Raffaello Mariani, and Jean-Michel Deluc in performing the wind-tunnel tests that developed and validated the grids: more generally, the involvement of the F1 and F2 teams during the wind-tunnel tests is acknowledged (especially the riggers for the grid reception and mounting) and the optical measurement staff for the setup and operation of the laser Doppler velocimetry and particle image velocimetry systems.

\section{References}

[1] Mouton, S., Masseboeuf, S., and Mariani, R., "Simulation of Atmospheric Boundary Layer Flow for Air Inlet Testing in F1 Wind-Tunnel," 33rd AIAA Aerodynamic Measurement Technology and Ground Testing Conference, AIAA Paper 2017-4132, June 2017. https://doi.org/10.2514/6.2017-4132 
[2] Seddon, J., and Goldsmith, E. L., Intake Aerodynamics, 2nd ed., AIAA Education Series, AIAA, Reston, VA, 1999, p. 266.

[3] Hall, C. A., and Humphreys, N. D., "Intake Performance During Rolling Take-Off in Natural Crosswind," 40th AIAA/ASME/SAE/ASEE Joint Propulsion Conference and Exhibit, AIAA Paper 2004-3403, July 2004. https://doi.org/10.2514/6.2004-3403

[4] Wakelam, C. T., Hynes, T. P., Hodson, H. P., Evans, S. W., and Chanez, P., "Separation Control for Aeroengine Intakes, Part 1: Low-Speed Investigation of Control Strategies," Journal of Propulsion and Power, Vol. 28, No. 4, 2012, pp. 758-765. https://doi.org/10.2514/1.B34326

[5] Wakelam, C. T., Hynes, T. P., Hodson, H. P., Evans, S.W ., and Chanez, P., "Separation Control for Aeroengine Intakes, Part 2: Low-Speed Investigation of Control Strategies," Journal of Propulsion and Power, Vol. 28, No. 4, 2012, pp. 766-772. https://doi.org/10.2514/1.B34327

[6] Colin, Y., Aupoix, B., Boussuge, J. F., and Chanez, P., "Numerical Simulation and Analysis of Crosswind Inlet Flows at Low Mach Numbers," Proceedings of the 8th International Symposium on Experimental and Computational Aerothermodynamics of Internal Flows, edited by I. Trébinjac, Paper ISAIF8-0058, Lyon, France, July 2007, https://hal.archives-ouvertes.fr/hal-03224309.

[7] Murphy, J. P., MacManus, D. G., and Sheaf, C. T., "Experimental Investigation of Intake Ground Vortices During Takeoff," AIAA Journal, Vol. 48, No. 3, March 2010, pp. 688-701. https://doi.org/10.2514/1.45896

[8] Carrara, J.-M., and Masson, A., "Three Years of Operation of the ONERA Pressurized Subsonic Wind Tunnel," 12th Congress of the International Council of the Aeronautical Sciences, ICAS, Bonn, Oct. 1980, pp. 778-792, https://hal.archives-ouvertes.fr/hal03224252 .

[9] Quémard, C., Garçon, F., and Raynal, J.-C., "High-Reynolds Number Air Intake Tests in the ONERA F1 and S1MA Wind-Tunnels," Workshop on Airframe Engine Integration at DLR Braunschweig, March 1996, https:// hal.archives-ouvertes.fr/hal-03224211.

[10] Hoelmer, W., Younghans, J. L., and Raynal, J.-C., "Effect of Reynolds Number on Upper Cowl Flow Separation," Journal of Aircraft, Vol. 24, No. 3, 1987, pp. 161-169. https://doi.org/10.2514/3.45411

[11] Armitt, J., and Counihan, J., "The Simulation of the Atmospheric Boundary Layer in a Wind Tunnel," Atmospheric Environment, Vol. 2, Pergamon, New York, 1968, pp. 49-71. https://doi.org/10.1016/0004-6981(68)90019-X

[12] Cermak, J. E., "Applications of Wind Tunnels to Investigation of Wind-Engineering Problems," AIAA Journal, Vol. 17, No. 7, 1979, pp. 679-690, https://doi.org/10.2514/3.61203

[13] Lawson, T. W., "Methods of Producing Velocity Profiles in Wind Tunnels," Atmospheric Environment, Vol. 2, Pergamon, New York, pp. 73-76. https://doi.org/10.1016/0004-6981(68)90020-6

[14] Counihan, J., "An Improved Method of Simulating an Atmospheric Boundary Layer in a Wind Tunnel," Atmospheric Environment, Vol. 3, Pergamon, New York, 1969, pp. 197-214. https://doi.org/10.1016/0004-6981(69)90008-0

[15] Irwin, H. P. A. H., "The Design of Spires for Wind Simulation," Journal of Wind Engineering and Industrial Aerodynamics, Vol. 7, No. 3, 1981, pp. 361-366. https://doi.org/10.1016/0167-6105(81)90058-1

[16] Ivanco, T. G., Keller, D. F., Pinkerton, J. L., Disotell, K. J., Collins, J. G., and Seliquini, S. L., "Development of an Atmospheric-Boundary-Layer Profile at the NASA Langley Transonic Dynamics Tunnel," 2018 AIAA Space and Astronautics Forum and Exposition, AIAA Paper 2018-5184, Sept. 2018. https://doi.org/10.2514/6.2018-5184

[17] Characteristics of Atmospheric Turbulence Near the Ground, Part I: Definitions and General Information, ESDU 74030, Engineering Science Data Unit, April 2010, https://www.esdu.com/.

[18] Characteristics of Atmospheric Turbulence Near the Ground, Part II: Single Point Data for Strong Winds (Neutral Atmosphere), ESDU 85020, Engineering Science Data Unit, Aug. 2001, https://www.esdu. com/.
[19] "Flying Qualities of Piloted Aircraft," U.S. Dept. of Defense MIL-STD1797A, Aug. 2004.

[20] Hall, C. A., and Hynes, T. P., "Nacelle Interaction with Natural Wind Before Takeoff," Journal of Propulsion and Power, Vol. 21, No. 5, 2005, pp. 784-791. https://doi.org/10.2514/1.2848

[21] Owen, P. R., and Zienkiewicz, H. K., "The Production of Uniform Shear Flow in a Wind Tunnel," Journal of Fluid Mechanics, Vol. 2, No. 6, 1957, pp. 521-531. https://doi.org/10.1017/S0022112057000336

[22] Elder, J. W., "Steady Flow Through Non-Uniform Gauzes of Arbitrary Shape," Journal of Fluid Mechanics, Vol. 5, No. 3, 1959, pp. 355-368. https://doi.org/10.1017/S0022112059000258

[23] Batchelor, G. K., The Theory of Homogeneous Turbulence, Cambridge Univ. Press, New York, 1967, pp. 58-68.

[24] Karnik, U., and Tavoularis, S., "Generation and Manipulation of Uniform Shear with the Use of Screens," Experiments in Fluids, Vol. 5, No. 4, 1987, pp. 247-254. https://doi.org/10.1007/BF00279737

[25] Van Atta, C. W., and Chen, W. Y., "Measurements of Spectral Energy Transfer in Grid Turbulence," Journal of Fluid Mechanics, Vol. 34, No. 3, 1968, pp. 497-515. https://doi.org/10.1017/S0022112068002041

[26] Uberoi, M. S., "Energy Transfer in Isotropic Turbulence," Physics of Fluids, Vol. 6, No. 8, Aug. 1963, pp. 1048-1056. https://doi.org/10.1063/1.1706861

[27] Rose, W. G., "Results of an Attempt to Generate a Homogeneous Turbulent Shear Flow," Journal of Fluid Mechanics, Vol. 25, No. 1, 1966, pp. 97-120. https://doi.org/10.1017/S0022112066000077

[28] Amandolèse, X., and Széchényi, E., "Experimental Study of the Effect of Turbulence of a Section Model Blade Oscillating in Stall," Journal of Wind Energy, Vol. 7, No. 4, 2004, pp. 267-282. https://doi.org/10.1002/we.137

[29] Afchain, D., Broussaud, P., Frugier, M., and Rancarani, G., "La Soufflerie F2 du Centre du Fauga-Mauzac (The F2 Wind-Tunnel of the FaugaMauzac Center)," 20ème Colloque d'Aérodynamique Appliquée AAAF, Paris, Association Aéronautique et Astronautique de France (AAAF), Nov. 1983; also NASA-TM-77482, 1984, https://hal archives-ouvertes.fr/hal-03224322.

[30] Rigo, F., Denoël, V., and Andrianne, T., "Vortex Induced Vibrations of Rectangular Cylinders Arranged on a Grid," Journal of Wind Engineering \& Industrial Aerodynamics, Vol. 177, June 2018, pp. 327-339. https://doi.org/10.1016/j.jweia.2018.04.011

[31] Masseboeuf, S., Mouton, S., Mariani, R., and Leclaire, B., "Clinometric Measurements by Means of High-Accuracy PIV in the ONERA F1 Low-Speed Pressurized Wind-Tunnel," 13th Asian Symposium on Flow Visualization, Inst. of Theoretical and Applied Mechanics, Novosibirsk, 2015.

https://doi.org/10.13140/RG.2.1.2041.2648

[32] Bousquet, J. M., Lyonnet, M., and Mignosi, A., "New Measurement Techniques in the Onera Large Wind-Tunnels," 25th Congress of the International Council of the Aeronautical Sciences, Vol. 3, ICAS, Bonn, 2006, pp. 1652-1660, https://hal.archives-ouvertes.fr/hal-03224281.

[33] Grandemange, M., Gohlke, M., and Cadot, O., "Turbulent Wake Past a Three-Dimensional Blunt Body. Part 1. Global Modes and Bi-Stability," Journal of Fluid Mechanics, Vol. 722, May 2013, pp. 51-84. https://doi.org/10.1017/jfm.2013.83

[34] Hall, C. A., and Hynes, T. P., "Measurements of Intake Separation Hysteresis in a Model Fan and Nacelle Rig," Journal of Propulsion and Power, Vol. 22, No. 4, 2006, pp. 872-879. https://doi.org/10.2514/1.18644

[35] Krone, J.-H., Harjes, L., Frantzheld, P., Koch, P., Giesecke, D., and Friedrichs, J., "Atmospheric Crosswind Tests of Aspirated Jet Engine Intake Models," Proceedings of Zürich 2019 Global Power and Propulsion Forum, Global Power \& Propulsion Soc. Paper GPPS-TC2019-0018, 2019. https://doi.org/10.33737/gpps19-tc-018

P. Lavoie Associate Editor 